Check for updates

Cite this: RSC Adv., 2018, 8, 6759

Received 23rd November 2017

Accepted 5th February 2018

DOI: $10.1039 / c 7 r a 12696 d$

rsc.li/rsc-advances

\section{Preparation of ferulic acid-grafted chitosan using recombinant bacterial laccase and its application in mango preservation}

\author{
Jie Yang, $\mathbb{D} \dagger$ Jianna Sun, $\uparrow$ Xiujuan An, $\uparrow$ Meixia Zheng, Zhaoxin Lu, Fengxia Lu \\ and Chong Zhang*
}

\begin{abstract}
To improve the antioxidant and antibacterial properties of chitosan, recombinant bacterial laccase from Bacillus vallismortis fmb-103 (fmb-rL103) was used to catalyze ferulic acid grafting. The grafted chitosan was characterized using UV-vis and FT-IR techniques. DPPH free radical scavenging results indicated that the antioxidant properties of the grafted chitosan (FA-g-CS) were significantly improved. Meanwhile, the antibacterial properties against E. coli, S. aureus, B. subtilis, and M. guilliermondii were also improved. Furthermore, FA-g-CS was applied to mango preservation as a coating, which improved the sensory qualities of mango Mangifera indica L. The disease incidence of mangoes coated with FA-grafted medium and high molecular weight chitosan were 0 and $5 \%$, respectively. The respiratory peak was delayed more than 4 days, and the titratable acidity and ascorbic acid concentration were all enhanced. POD and CAT activities in FA-g-CS coated mangoes were higher than those coated with chitosan samples with a lower $\mathrm{H}_{2} \mathrm{O}_{2}$ concentration.
\end{abstract}

\section{Introduction}

Chitosan (CS), the most abundant natural amino polysaccharide, is widely used in food preservation. ${ }^{1}$ Grafting CS with small molecules can improve its antioxidant and antibacterial activities. Many phenolic antioxidants, including gallic, caffeic, and ferulic acids (FA) have been grafted onto CS to improve these properties. $^{2}$ Recently, an enzyme-catalyzed grafting method has been used for the synthesis of phenolic acid-g-chitosan that has mild reaction conditions, high reaction efficiency, fewer byproducts, and is environmentally friendly. ${ }^{3,4}$

Laccases (benzenediol:oxygen oxidoreductases; EC 1.10.3.2) was first found in the exudates of Japanese lacquer trees and have since been found in many other species including insects, plants, archaea, and bacteria. ${ }^{5}$ Some studies have reported the grafting of phenolic compounds onto CS using fungal laccase through Michael addition or Schiff base reactions. Aljawish et al. used Myceliophthora thermophila laccase to catalyze the grafting of ferulic acid or ethyl ferulate onto CS, affording materials with improved antioxidant and antibacterial properties. ${ }^{6,7}$ Božič et al. ${ }^{8}$ also reported that laccase from Trametes versicolor can catalyze the grafting of caffeic and gallic acids onto CS. Qiu et al. ${ }^{9}$ have reported the laccase catalyzed the grafting of tea catechins onto CS using laccase to prepare

Laboratory of Enzyme Engineering, College of Food Science and Technology, Nanjing Agricultural University, Nanjing 210095, P. R. China. E-mail: zhangchong@njau. edu.cn; Fax: +86-25-84396583; Tel: +86-25-84396521

$\dagger$ Co-first author. a novel nanofiltration membrane (obtained from SigmaAldrich, fungus source). Although, fungal laccases can catalyze grafting reactions, their activity usually decays rapidly at high temperatures and $\mathrm{pH}$ values, while bacterial laccases have comparatively good temperature and $\mathrm{pH}$ stability. However, to our knowledge, the catalysis of phenolic compound grafting onto CS through bacterial laccase has not been reported.

In our previous study, to enhance laccase productivity, the laccase gene from B. vallismortis strain fmb-103 was cloned and efficiently expressed in $E$. coli using an autoinduction expression strategy. Fmb-rL103 has a high optimum temperature, is thermostable, and is $\mathrm{pH}$ stable, especially under acidic $\mathrm{pH}$ conditions. ${ }^{10}$ This stability makes fmb-rL103 a promising candidate for potential application to grafting FA onto CS.

Mango (Mangifera indica L.) is a tropical fruit popular for its attractive shape, fragrance, and nutritional properties. ${ }^{11}$ However, mango is a typical climacteric fruit that quickly ripens and softens after harvest. Maturation can be accelerated by ethylene and the fruits are prone to chilling injury, anthracnosis, and stem-end rot. ${ }^{12}$ Coating is a simple, low cost, and secure method for extending the commercial shelf-life of fruits. ${ }^{13}$ Coating can reduce fruit water loss and change the composition of the atmosphere surrounding the fruit to delay ripening and softening. Furthermore, coating can maintain the fruit quality by inhibiting fungal growth, which improves the appearance during transportation and storage. ${ }^{14}$

The present study aims to establish an efficient method for grafting FA onto CS using bacterial laccase, and to study improvements in the properties of FA-g-CS, particularly its 
antioxidant and antibacterial activities. FA- $g$-CS was then applied to mango preservation to improve storage quality.

\section{Results \& discussion}

\section{Synthesis of FA- $g$-CSs using recombinant bacterial laccase}

To synthesize FA- $g$-CSs effectively, the effects of $\mathrm{pH}$, temperature, time, and FA concentration on the grafting reaction were investigated by measuring the degree of FA substitution onto CS.

Fig. 1A shows the pH effect on the degree of FA substitution. When the $\mathrm{pH}$ was 4.5 , the protonation of the primary amino groups resulted in CS having no nucleophilicity, resulting in a lowest degree of FA substitution. ${ }^{15,16}$ When the $\mathrm{pH}$ was 5.5, some amino groups on CS were protonated, such that only a small amount (about 9\%) of the amino groups could participate in the substitution reaction. ${ }^{15}$ At $\mathrm{pH}$ 6.5, less amino groups on CS were protonated, and the small amount of $\mathrm{H}^{+}$present under weak acid conditions contributed to the substitution reaction. However, at pH 7.5, a lower degree of FA substitution were achieved, probably due to the low activity, poor stability, and weak catalytic capacity of laccase at $\mathrm{pH} 7.5$, and the lack of
$\mathrm{H}^{+}$assisting the reaction under these weakly alkaline conditions. Therefore, the grafting reaction rate and degree of FA substitution was highest at $\mathrm{pH}$ 6.5.

Fig. 1B shows the effect of reaction time on the degree of FA substitution. In the initial stages of the reaction $(0-1 \mathrm{~h})$, fmbrL103 catalyzed FA and the catalytic product adsorbed to CS, followed by a grafting reaction. During this process, many amino groups on CS were exposed, making it easier for the catalytic products of FA to adsorb to CS and undergo grafting reactions. The degree of FA substitution increased rapidly. As the reaction progressed, amino groups on CS were gradually grafted and the number of reaction sites decreased. Furthermore, FA was almost completely consumed in the reaction system, the grafting reaction rate rapidly decreased, and the amino group content started to increase, probably because the catalysis reaction consisted of two reversible processes, namely polymerization and depolymerization. The polymerization rate was much higher than that of depolymerization within $1 \mathrm{~h}$, resulting in a higher reaction rate. After $1 \mathrm{~h}$, the polymerization rate slowed and the depolymerization rate increased, leading to a decrease in amino groups. When the catalysis reaction was carried out at a higher temperature $\left(60^{\circ} \mathrm{C}\right)$, the highest
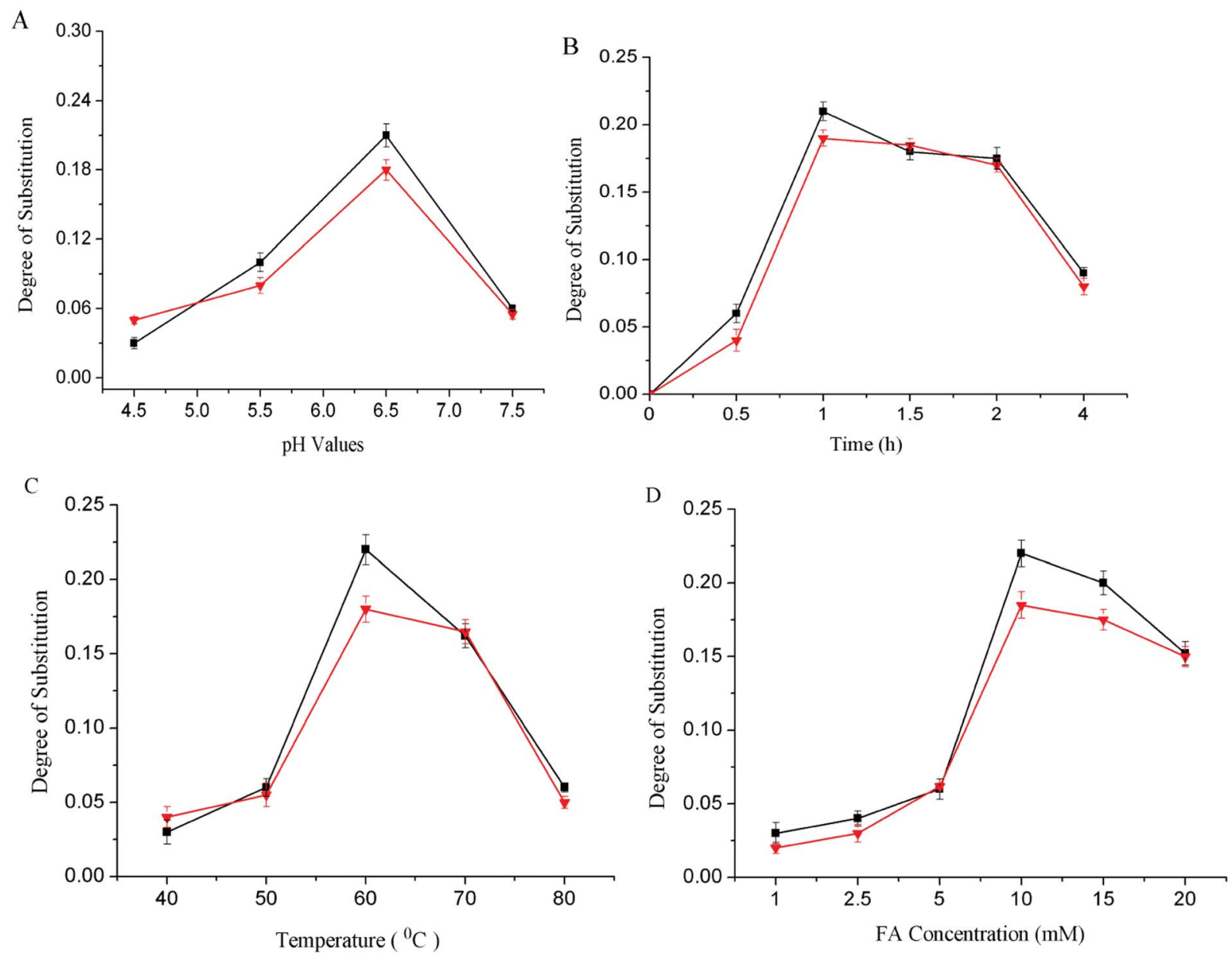

Fig. 1 Effect of (A) pH, (B) time, (C) temperature, and (D) substrate concentration on the reaction of FA-grafted CSs: FA-g-HMW CS (black, and FA-g-MMW CS (red, $\boldsymbol{\nabla}$ ). Data are presented as means \pm SD of triplicates. 
substitution was reached after $1 \mathrm{~h}$. Compared with previously reported experiments, this represented a significant reduction in reaction time. ${ }^{16}$

The effects of reaction temperature on the grafting reaction were studied under the following conditions: $\mathrm{pH}$ 6.5; time, $1 \mathrm{~h}$; and FA concentration, $10 \mathrm{mM}$. As shown in Fig. 1C, the FA substitution degree was highest at $60{ }^{\circ} \mathrm{C}$. When the concentrations of CS and FA were constant, the amino group content decreased with increasing temperature in the range $30-60{ }^{\circ} \mathrm{C}$, showing a particularly rapid decrease at $50-60{ }^{\circ} \mathrm{C}$. The grafting reaction involves two reversible processes, polymerization and depolymerization, of which the polymerization reaction rate was higher than the depolymerization reaction rate at low temperature. While, at $60{ }^{\circ} \mathrm{C}$, the polymerization reaction rate was equal to that of the depolymerization reaction, and the grafting reaction rate and FA substitution degree were highest.

The effect of FA concentration on the grafting reaction is shown in Fig. 1D. The FA substitution degree of CS was highest at an FA concentration of $10 \mathrm{mM}$. At a low FA concentration, the amount of FA products catalyzed by fmb-rL103 was lower, resulting in a low adsorption and grafting reaction rate with CS. An FA concentration of $10 \mathrm{mM}$ ensured FA oxidation and grafting of the catalytic products onto CS. When the FA concentration was higher than $10 \mathrm{mM}$, the large amount of FA molecules might interfere with the adsorption and grafting reactions of the catalytic products with $\mathrm{CS}$, resulting in a reduced grafting efficiency.

Therefore, under the optimal reaction conditions $(\mathrm{pH} 6.5$; reaction time $1 \mathrm{~h}$; temperature, $60^{\circ} \mathrm{C}$; fmb-rL103 concentration, $3 \mathrm{U} \mathrm{mL}^{-1}$ ) the FA substitution degrees of high and medium molecular weight CSs were 0.192 and 0.225 , respectively. In comparison, the CS grafting derivatives prepared using fungal laccase required more enzyme (13.5 $\left.\mathrm{U} \mathrm{mL}^{-1}\right)$ and a longer reaction time $(4 \mathrm{~h})$, while fmb-rL103 showed much better grafting efficiency and practicality. ${ }^{6}$

\section{UV/vis and FT-IR analyses}

The FA-grafted CSs (HMW and MMW) prepared using fmbrL103 had a stable orange color. Further characterization was performed on the CSs and FA- $g$-CSs to confirm the grafting reaction. Significant changes in the UV/vis spectra were observed, as shown in Fig. 2A. The large increase in absorbance at 300-350 $\mathrm{nm}$ was probably due to a reaction between the free radical products of FA catalyzed by fmb-rL103 and the free amino groups of CSs. ${ }^{17}$ Furthermore, FA- $g$-CSs absorbed in the visible region, while the three control groups showed no absorption. These results indicated that laccase-catalyzed oxidized FA could be covalently grafted onto CSs.

As shown in the FT-IR spectra (Fig. 2B), the absorption bands of grafted chitosan at $1420 \mathrm{~cm}^{-1}$ (symmetric $-\mathrm{NH}_{3}{ }^{+}$bending region), $1380 \mathrm{~cm}^{-1}$, and $1320 \mathrm{~cm}^{-1}(\mathrm{~N}-\mathrm{H}$ bending of glucosamine unit) were decreased, which indicated the loss of $\mathrm{NH}_{3}{ }^{+}$ groups and a covalent reaction between the products of laccasecatalyzed FA and the CS amino groups. ${ }^{17}$ Additionally, the decrease in absorption at $1420 \mathrm{~cm}^{-1}$ might be related to the protonated glucosamine residues of CS. The change at 1000-
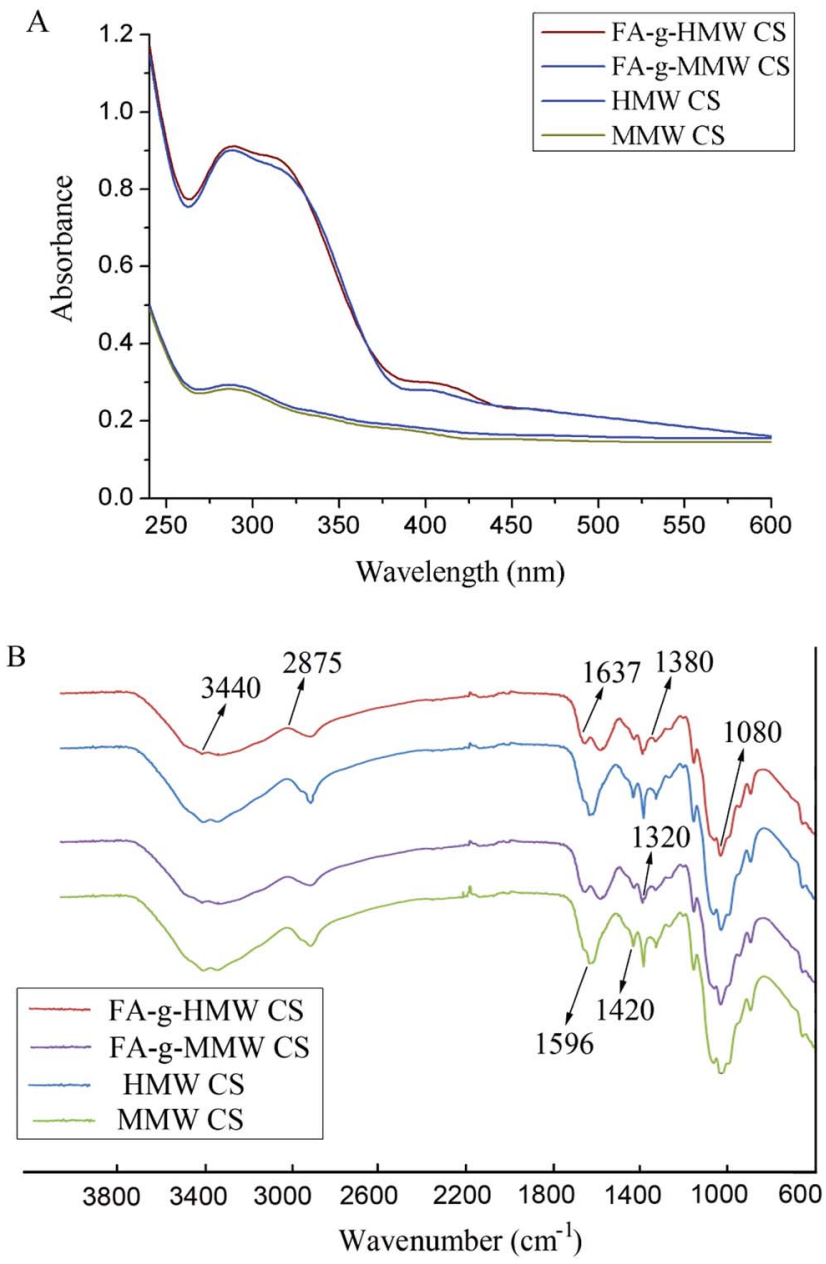

Fig. 2 (A) UV-vis and (B) FT-IR spectra of CSs and FA-g-CSs.

$1500 \mathrm{~cm}^{-1}$ might be related to aromatic $\mathrm{C}-\mathrm{O}$ and aliphatic $\mathrm{C}-\mathrm{O}$ bond stretching in $\mathrm{FA}^{\mathbf{1 8}}$ and the disappearance of the absorption at $1596 \mathrm{~cm}^{-1}$ could indicate the decrease in amino groups on CS. New bands appeared at $1637 \mathrm{~cm}^{-1}$, corresponding to $\mathrm{C}=\mathrm{N}$ vibrations that were characteristic of imines and $\mathrm{C}=\mathrm{C}$ stretching vibrations due to Schiff bases formed between the amino groups of the CS pyranose cycle and phenolic moieties. ${ }^{19}$ These changes in the spectra verified that the amino groups on CS were subjected to Schiff-base-type covalent modifications by the products of laccase-catalyzed FA.

These results suggested that fmb-rL103-catalyzed grafting of FA onto CSs could be divided into two steps. First, fmb-rL103 oxidizes FA to an active free radical, and the FA oxidation product is then covalently bound with an amino group at the CS C-2 position through a Schiff base addition reaction.

\section{Antioxidant properties}

The stable DPPH system is usually used to determine the free radical scavenging activity of antioxidants. Fig. 3 shows that, for both MMW and HMW CS derivatives, the antioxidant activity of grafted CS were significantly increased compared with ungrafted CS. At a concentration of $2.0 \mathrm{mg} \mathrm{mL}^{-1}$, the DPPH free 


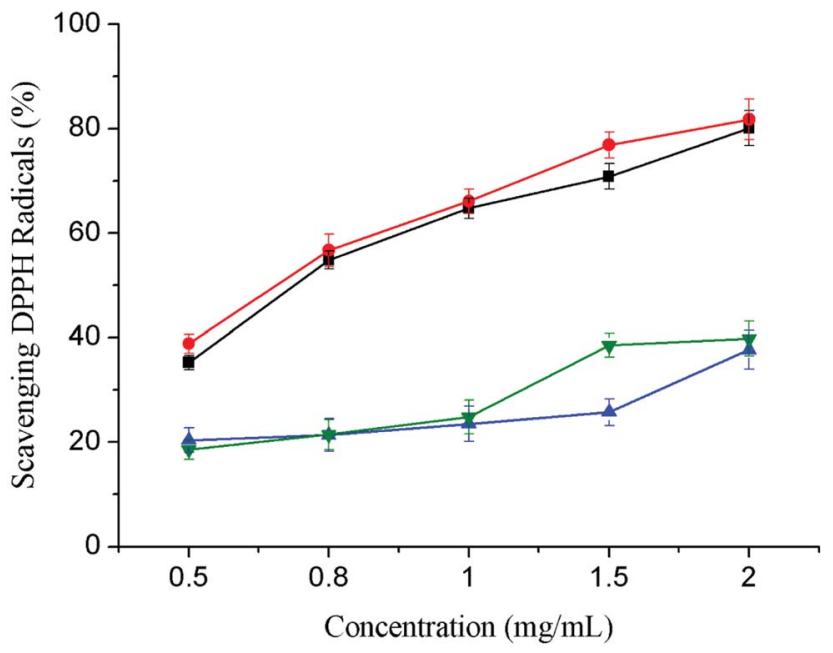

Fig. 3 Scavenging activities of FA- $g$-CSs on DPPH radicals: FA- $g$ HMW CS (black, $\boldsymbol{\square}$ ), FA-g-MMW CS (red, - HMW CS (blue, $\boldsymbol{\Delta}$ ), and MMW CS (green, $\boldsymbol{\nabla})$. Data are presented as means \pm SD of triplicates.

radical scavenging rate of MMW FA- $g$-CS was $81.79 \%$, with an $\mathrm{EC}_{50}$ value of $0.65 \pm 0.02 \mathrm{mg} \mathrm{mL}{ }^{-1}$. The $\mathrm{DPPH}$ free radical scavenging rate of HMW FA- $g$-CS was $80.08 \%$, with an $\mathrm{EC}_{50}$ value of $0.71 \pm 0.03 \mathrm{mg} \mathrm{mL}^{-1}$. Meanwhile, the DPPH free radical scavenging rates of MMW and HMW CS were less than $40 \%$, respectively.

\section{Antibacterial activity}

Table 1 shows the antibacterial activity of the samples against $E$. coli ATCC 25922, S. aureus ATCC 25923, B. subtilis ATCC 168, and M. guilliermondii. Compared with the CS, the FA- $g$-CS showed a higher antibacterial activity against these four indicator bacteria. At a concentration of $1 \mathrm{mg} \mathrm{mL}^{-1}$, the antibacterial ring of MMW FA- $g$-CS was increased by 2.09, 1.87, 1.38, and $1.44 \mathrm{~mm}$, respectively, while the antibacterial ring of HMW FA- $g$-CS was increased by $2.19,1.50,1.68$, and $1.48 \mathrm{~mm}$, respectively.

However, these results showed that the antibacterial activity of CS and FA- $g$-CS did not increase with increasing concentration (data not shown). This was probably because the polycation of CS more easily adsorbed and combined with bacteria at low concentration, while a high concentration of CS might lead to stabilization. At low concentrations, CS or its derivatives may change the cell membrane permeability, blocking nutrient entry and leading to cell death. However, high concentrations of CS or its derivatives can densely wrap the cell surface, reducing cytoplasmic leakage.

\section{Effects of FA- $g$-CS coating on mango}

Percentage of weight loss, firmness, and disease incidence of mango. Water is lost from mango fruit due to the dual effects of respiration and transpiration, which affect the mango appearance, including color, firmness, and weight, and shorten the storage life.$^{20}$ A CS or FA- $g$-CS coating can significantly delay the yellowing process and increase the peel luster (Fig. 4). The uncoated fruit began to turn yellow on day 8 of storage, while part of the fruit peel had undergone kraurosis and showed a higher weight loss (Table 2). In contrast, the coating treatment groups showed no changes in color on day 16, with a higher hardness and lower weight loss. As shown in Table 2, the FA- $g$ CS coating resulted in better firmness and lower weight loss than the CS coating.

Table 2 also shows that the coating treatment had a significant effect on the disease incidence. The disease incidence of the control group was 55\% after 16 days, while the disease incidence of the coated groups was $\leq 15 \%$. In particular, the disease incidence of FA- $g$-MMW CS was 0 on day 16, compared with 15\% for MMW CS.

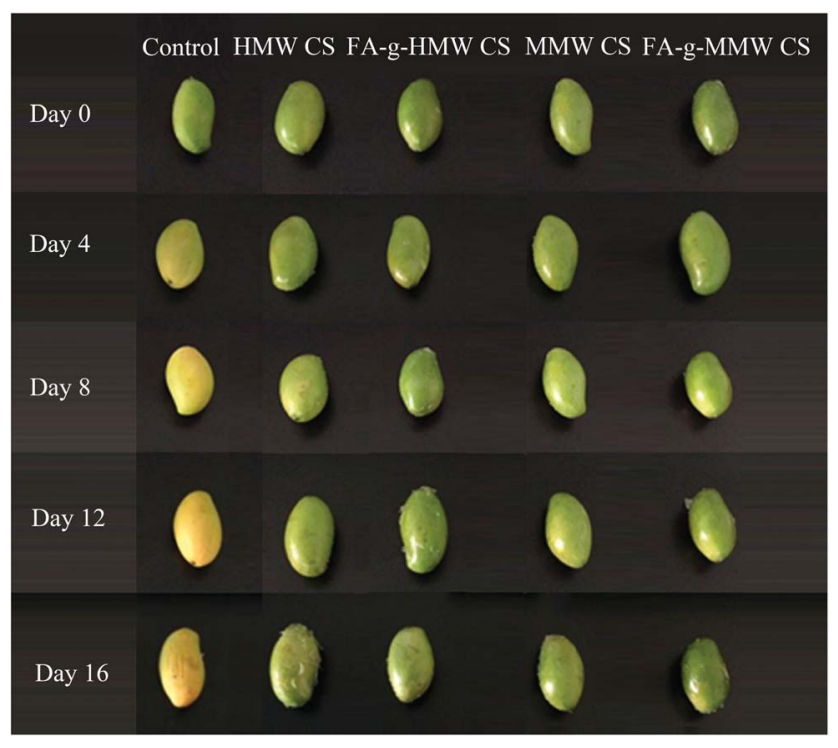

Fig. 4 Mango appearance after coating with CS and FA-g-CS during storage at $25^{\circ} \mathrm{C}$ for 16 days.

Table 1 The antimicrobial activities of CS and grafted CS

\begin{tabular}{|c|c|c|c|c|}
\hline \multirow[b]{2}{*}{ Sample } & \multicolumn{4}{|c|}{ Bacteriostatic ring (mm) } \\
\hline & E. coli & Staphylococcus aureus & Bacillus subtilis & Yeast \\
\hline MMW CS & $14.69 \pm 0.19$ & $15.79 \pm 0.35$ & $15.67 \pm 0.14$ & $13.48 \pm 0.22$ \\
\hline FA- $g$-MMW CS & $16.78 \pm 0.13$ & $17.66 \pm 0.03$ & $17.05 \pm 0.10$ & $14.92 \pm 0.16$ \\
\hline HMW CS & $14.84 \pm 0.15$ & $16.14 \pm 0.17$ & $15.44 \pm 0.20$ & $13.66 \pm 0.19$ \\
\hline FA- $g$-HMW CS & $17.03 \pm 0.20$ & $17.64 \pm 0.32$ & $17.12 \pm 0.11$ & $15.14 \pm 0.34$ \\
\hline
\end{tabular}


Table 2 Qualities changes of mango during storage at $25^{\circ} \mathrm{C}$ for 16 days

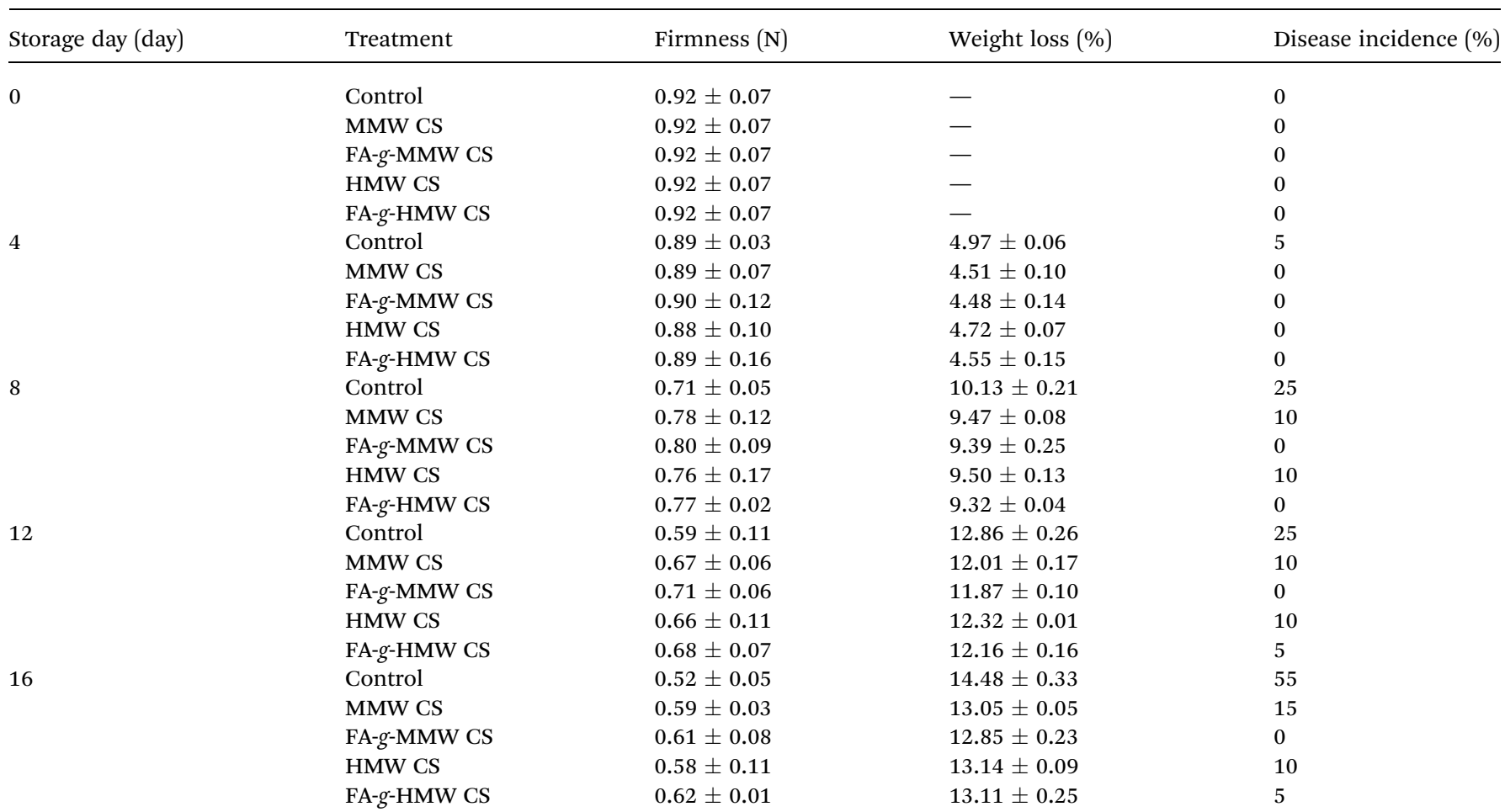

The CS coatings can change the composition of the atmosphere surrounding the fruits, which can inhibit softening and ripening. The coating can also act as an exogenous stimulating factor to induce activities associated with defense-related enzymes and accumulate some of the plant's special substances, participate in defense mechanisms and prevent pathogen infection, achieving a preservation effect. ${ }^{21}$ Notably, the disease incidence of the CS coating groups was higher than that of FA- $g$-CS treatment samples, due to the enhanced antibacterial properties of FA- $g$-CSs. The FA- $g$-CS film coatings on mango were more uniform, dense, and effective at reducing the fruit oxygen permeability and water permeability than the CS coatings, resulting in inhibited fruit respiration and transpiration.

Respiration rate, total soluble solids, titratable acidity and ascorbic acid content. Respiration rate is an important index for evaluating physiological changes in postharvest fruit, and can reflect the nutrient consumption and aging of fruits and vegetables during storage. ${ }^{22}$ Fig. $5 \mathrm{~A}$ shows that the respiration of uncoated mango reached a peak on day 8 , while the coating groups reached peak respiration on day 12 . Compared with the control group, the respiration rates of the CS and FA-g-CS groups were delayed and lower within 12 days. Furthermore, mangoes coated with FA-g-CSs had lower respiration rates than those coated with CSs. This was probably due to the FA-g-CS films being denser, which reduced the fruit oxygen permeability and water permeability.

The soluble solids content of the control group increased and decreased slowly (Fig. 5B). It showed a downward trend on the 8th day, and decreased to $18.82 \%$ on the 16 th day. The soluble solids content of coating treatment groups increased rapidly in 0-12 days, and showed a downward trend on the 12th day, with the highest content of $21.13 \%$ on the 16 th day (FA-g-CS coating treatment), indicating that chitosan coating can delay the fruit repenting.

TA is among the primary flavors of mango fruit and an important indicator of fruit maturity. ${ }^{22}$ TA can inhibit the growth of microorganisms, and reduce the susceptibility of mangoes to bacterial infections. Furthermore, organic acids have antioxidant properties that protect ascorbic acid from damage and maintain mango color. However, organic acid can be gradually metabolized into carbon dioxide and water during storage. Due to consumption by respiration, the titratable acidity of mango decreased with storage time. As shown in Fig. 5C, the titratable acidity content of uncoated group decreased rapidly in the early stages of storage, and the rate of decrease was significantly lower after day 8 , probably due to the respiration and acid consumption becoming slower. Throughout the storage period, the FA-g-CS-coated samples had the lowest titratable acid reduction rates at each determination time point. This showed that the FA- $g$-CS coatings more effectively delayed the degradation and transformation of titratable acid than CS coatings, and maintained a high acid content in the mango.

Ascorbic acid is not only a primary nutritional component in mango, but also an important antioxidant in fruit. ${ }^{23}$ However, it is easily oxidized by ascorbic acid oxidase during storage. Fig. 5D shows that the ascorbic acid content of mango 

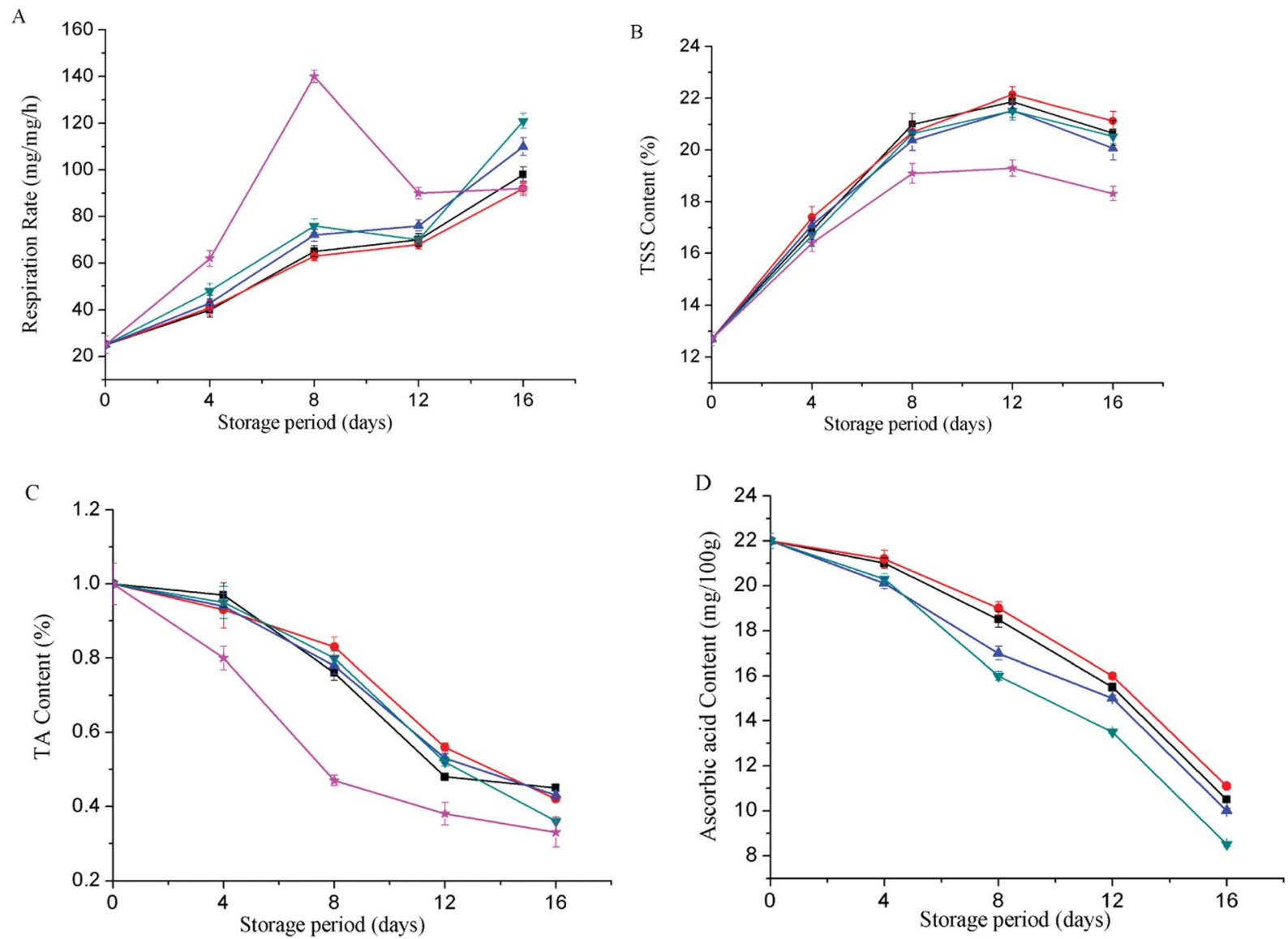

Fig. 5 Effect of coating with CS and FA-g-CS on (A) respiration rate, (B) TSS, (C) TA and (D) ascorbic acid content of mangoes. FA-g-HMW CS (black, $\mathbf{\square})$, FA-g-MMW CS (red, - ), HMW CS (blue, $\mathbf{\Delta}$ ), MMW CS (green, $\boldsymbol{\nabla}$ ) and control (pink, $\star$ ). Data are presented as means \pm SD of triplicates.

decreased with increasing storage time. By day 16 , the ascorbic acid content of the control group was only $6.4 \mathrm{mg} / 100 \mathrm{~g}$, while that of coated mango was clearly higher. The sample coated with FA-g-MMW CS had the highest ascorbic acid content of $11.1 \mathrm{mg} / 100 \mathrm{~g}$ on day 16 . This indicated that coating with CS derivatives was more effective at maintaining a low $\mathrm{O}_{2}$ and high $\mathrm{CO}_{2}$ environment in mango fruit to inhibit ascorbic acid oxidase activity and reduce ascorbic acid decomposition.

POD, CAT activities and $\mathrm{H}_{2} \mathrm{O}_{2}$ content. Fruit ripening and aging can be considered an oxidation phenomenon. The production of reactive oxygen species increases and antioxidant activity decreases during fruit ripening. CS can increase the scavenging capacity of reactive oxygen species in fruits..$^{24,25}$

Peroxidase (POD) is an important antioxidant enzyme in fruit that can scavenge reactive oxygen species and inhibit fruit cell membrane damage. The POD activity can be used to assess the process of fruit senescence. As shown in Fig. 6A, the POD activity increased rapidly initially, and then decreased gradually during storage. The POD activity of the FA-g-CS-coated samples was higher than that of the CS-coated samples.

CAT can catalyze $\mathrm{H}_{2} \mathrm{O}_{2}$ decomposition and reduce the toxicity of reactive oxygen species to cells. The CAT activity decreased during storage. As shown in Fig. 6B, the CAT activities of mangoes treated with CS derivatives were higher than those of other groups. Early in storage (on day 4), the CAT activity of the samples coated with CS was about $130 \mathrm{U}$, while those of the samples coated with FA- $g$-CS (FA- $g$-MMW CS and FA-g-HMW CS) remained at about $150 \mathrm{U}$. On day 16, the CAT activity of the CS-coated groups was about $80 \mathrm{U}$, while both FA-gCS-coated groups remained above $100 \mathrm{U}$. These results indicated that FA- $g$-CSs were more beneficial for producing antioxidant enzymes and prolonging shelf life in mangoes.

The $\mathrm{H}_{2} \mathrm{O}_{2}$ content of mango coated with FA- $g$-CS was lower than that of the CS-coated and control groups (Fig. 6C). Later in storage, the $\mathrm{H}_{2} \mathrm{O}_{2}$ content of FA-g-CS-coated mango gradually became lower than that of the CS-coated mango, probably due to the increased antioxidant activity of FA- $g$-CS.

\section{Experimental}

\section{Bacterial laccase and other chemicals}

Temperature and pH-stable recombinant bacterial laccase (fmb-rL103) was prepared through heterologous production of laccase from Bacillus vallismortis fmb-103 in E. coli, and purified using a heating and ammonium sulfate dialyzing method, as described previously. ${ }^{10}$

FA was purchased from Sigma-Aldrich Chemical Co. (St. Louis, MO). CS HMW (high molecular weight, $400 \mathrm{kDa}$, 85\% 
A
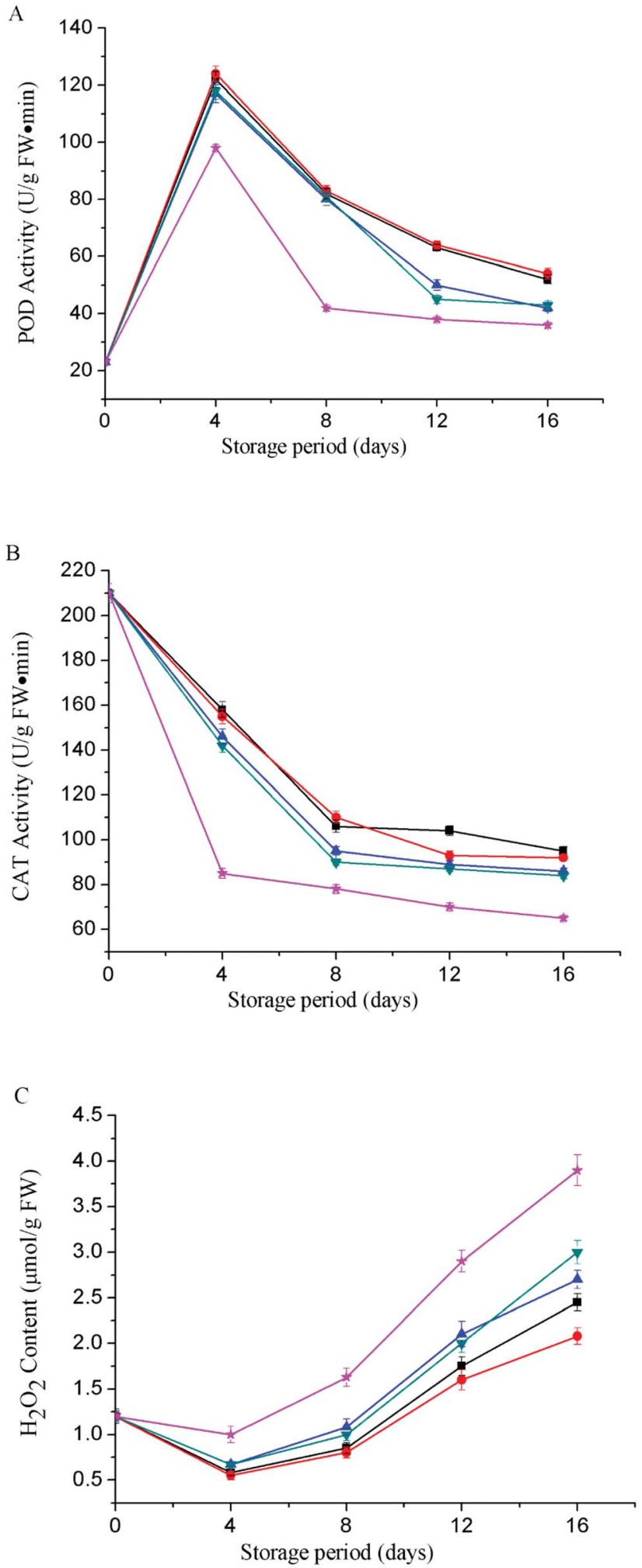

Fig. 6 Effect of coating with CS and FA-g-CS on (A) POD activity, (B) CAT activity TA, and (C) $\mathrm{H}_{2} \mathrm{O}_{2}$ content of mango. FA-g-HMW CS (black, 口), FA-g-MMW CS (red, - ), HMW CS (blue, $\mathbf{\Delta}$ ), MMW CS (green, $\mathbf{\nabla}$ ) and control (pink, $\star$ ). Data are presented as means \pm SD of triplicates.

deacetylation) and MMW (middle molecular weight, $150 \mathrm{kDa}$, $85 \%$ deacetylation) were purchased from Yongsheng Biotechnology Co., Ltd. (Shanghai, China). Mango (Mangifera indica L.) was purchased at a fruit market in Nanjing, China. All other conventional reagents were of analytical grade.

\section{FA- $g$-CS preparation}

Enzymatic synthesis of FA-g-CS was performed using a heterogeneous grafting method. CS powder $(1 \mathrm{~g})$ was dispersed in buffer solution $(45 \mathrm{~mL})$, followed by FA in methanol $(5 \mathrm{~mL}$, various concentrations) and fmb-rL103 $\left(3 \mathrm{U} \mathrm{mL}^{-1}\right)$.

The reaction solution was kept at $60{ }^{\circ} \mathrm{C}$ for $1 \mathrm{~h}$ with continuous stirring $(100 \mathrm{rpm})$. The solution was then cooled to room temperature and pellets were collected by centrifugation $(8000$ $\times g, 15 \mathrm{~min}, 4^{\circ} \mathrm{C}$ ). To remove ungrafted FA, the collected pellets were washed sequentially with methanol, ethanol, and water. Meanwhile, the ethanol that used for washing the collected pellets was checked by HPLC, to ensure the FA-g-CS not contains unbound ferulic acid. The products were then dried by vacuum freeze drying and stored at $4{ }^{\circ} \mathrm{C}$ for the remaining analysis.

The effects of $\mathrm{pH}$, temperature, reaction time, and FA concentration on the grafting efficiency were also studied. All experiments were performed in triplicate.

\section{Determination of grafting efficiency}

The grafting efficiency was measured using amino group substitution. The amino group contents of CS and FA- $g$-CS were determined based on a reported method with slight modification. ${ }^{26}$ CS and FA- $g$-CS were dissolved in acetic acid solution $(1 \%, \mathrm{v} / \mathrm{v})$ at a concentration of $1 \mathrm{mg} \mathrm{mL}{ }^{-1}$. The acetate buffer (pH 5.5, $0.5 \mathrm{~mL}$ ) and freshly prepared hydrated ninhydrin (50 $\mathrm{mg} \mathrm{mL} \mathrm{m}^{-1}$, ninhydrin dissolved in dimethylformamide, 2 $\mathrm{mL}$ ) was added to the sample solution. The mixture was heated in boiling water for $30 \mathrm{~min}$ and cooled to room temperature for measurement at $570 \mathrm{~nm}$. The lower absorbance value indicated the lower remaining amino content, and the degree of amino substitution was determined.

\section{UV-vis and FT-IR analysis}

CS and FA- $g$-CS were dissolved in acetic acid solution $(1 \%, \mathrm{v} / \mathrm{v})$ at a concentration of $1 \mathrm{mg} \mathrm{mL} \mathrm{m}^{-1}$ and subjected to full-wavelength scanning at $240-600 \mathrm{~nm}$ using a UV-visible (UV-vis) spectrophotometer (Shimadzu, Kyoto, Japan). FA-g-CS was analyzed by observing changes in characteristic peaks.

The samples were subjected to Fourier transform infrared spectroscopy (FT-IR) (Nicolet, Madison, WI) using 128 scans, scanning at $10 \mathrm{kHz}$ in the range $500-4000 \mathrm{~cm}^{-1}$. The ambient gas was air.

\section{Antioxidant activity}

CSs and FA- $g$-CS were dissolved in acetic acid solution (1\%, v/v) at concentrations of $0.5,0.8,1,1.2$, and $2 \mathrm{mg} \mathrm{mL}^{-1}$. A methanolic solution $(2 \mathrm{~mL})$ containing 1,1-diphenyl-2-picrylhydrazyl (DPPH) radicals $(2 \mathrm{mM})$ was added to $2 \mathrm{~mL}$ of CS solution. The mixture was shaken for $15 \mathrm{~s}$, and then left to stand in the dark at $33{ }^{\circ} \mathrm{C}$ for $30 \mathrm{~min}$. The absorbance of the resulting solution $\left(A_{\text {sample }}\right)$ was measured at $517 \mathrm{~nm}$ using UV-vis 
spectrophotometry. The control absorbance, $A_{\text {control, was }}$ measured using acetic acid solution (1\%, v/v) instead of a sample solution. The free radical scavenging activity was calculated using the following equation:

DPPH scavenging ability $(\%)=\left(1-A_{\text {sample }} / A_{\text {control }}\right) \times 100 \%$

The $\mathrm{EC}_{50}$ value, which expresses the antioxidant capacity of the sample, is the antioxidant concentration that reduced the number of radicals by $50 \%$.

All experiments were performed in triplicate.

\section{Antibacterial activity}

The antibacterial activity of CS and FA-g-CS against Escherichia coli (ATCC 25922), Staphylococcus aureus (ATCC 25923), Bacillus subtilis (ATCC 168), and Meyerozyma guilliermondii (isolated from mango by an enzyme engineering laboratory and identified based on the complete 26S rDNA D1/D2 gene sequences) were determined using the Oxford Cup method. Solutions of CS and FA- $g$-CS were prepared by dissolving in $0.1 \mathrm{M}$ hydrochloric acid. The indicated culture $\left(0.1 \mathrm{~mL}\right.$; bacterial concentration, $10^{5}$ to $10^{6} \mathrm{CFU} \mathrm{mL}{ }^{-1}$ ) was added to the medium plate. After evenly coating and drying for $30 \mathrm{~min}$, four disinfected sterilized Oxford cups were placed on each plate, and $0.2 \mathrm{~mL}$ of the sample solution was added. The plates were then incubated in an incubator and the diameters of bacteriostatic ring were determined.

\section{Spray treatment of mango}

120 mangoes were selected for their uniformity in size, color, shape, and lack of blemishes and disease symptoms. CS and FA$g$-CS $(10 \mathrm{~g}$ each) were separately dissolved in $600 \mathrm{~mL}$ of $1 \%$ acetic acid solution and sprayed onto the mango, which was then placed under constant temperature and humidity. After treatment, the fruit was air-dried for $30 \mathrm{~min}$ and stored at $25^{\circ} \mathrm{C}$.

\section{Percentage of weight loss, firmness, and disease incidence of mango}

The weight loss was calculated as a percentage loss from the initial weight. The mangoes were weighed regularly to determine weight loss using the formula:

$$
\text { Weight loss }(\%)=\left(m_{1}-m_{2}\right) / m_{1} \times 100
$$

where $m_{1}$ is the initial fruit weight and $m_{2}$ is the fruit weight at the time of detection. ${ }^{27}$

The firmness was evaluated using a hardness tester in the same three regions as used for peel color measurements. The firmness was recorded as a $\mathrm{kg}$ force in newtons $(\mathrm{N})$.

Disease incidence was measured as a percentage by counting the diseased fruit and calculated as follows:

Disease incidence $(\%)=$ no. of diseased fruits/no. of total fruits $\times 100 \%$

\section{Respiration rate}

A stationary method was used to determine the mango respiration rate. ${ }^{28} \mathrm{NaOH}$ solution $(20 \mathrm{~mL})$ was placed into a culture dish. The culture dish and mango were placed into a prepared dryer and sealed for $1 \mathrm{~h}$. After removing the culture dish, the solution was dissolved in an iodine bottle and saturated $\mathrm{BaCl}_{2}$ solution ( $5 \mathrm{~mL}$ ) and 2 drops of $1 \%$ phenolphthalein were added. The solution was titrated with $0.2 \mathrm{M}$ oxalic acid solution until the solution was discolored and did not fade within $30 \mathrm{~s}$. The same method was used for the control group. The respiration rate was calculated as follows:

$$
\text { Respiration rate }=\left(V_{1}-V_{2}\right) \times M \times 44 W \times h \times 100 \%
$$

where $M$ is the oxalic acid concentration (M), $V_{1}$ is the titration volume of the control group (mL), $V_{2}$ is the titration volume of the sample $(\mathrm{mL}), W$ is the sample weight $(\mathrm{kg}), h$ is the determination time, and 44 represents the molecular weight of $\mathrm{CO}_{2}$.

\section{Total soluble solids content}

The mango pulp was ground and filtered with four layers of gauze, and the juice was used to determine total soluble solids with a hand refractometer.

\section{Titratable acidity content}

The titratable acidity (TA) analysis method was applied from Jongsri. ${ }^{29}$ One hundred $\mathrm{mL}$ of distilled water was mixed with $10 \mathrm{~g}$ of sliced mango pulp by vortex for $1 \mathrm{~min}$ and filtered. $10 \mathrm{~mL}$ of filtrate was titrated with $0.1 \mathrm{M} \mathrm{NaOH}$. Filtrate was added with $1 \%$ phenolphthalein indicator; then titrated until the end point. The TA (\%) was calculated as follows:

$$
\mathrm{TA}(\%)=\frac{\mathrm{NaOH}(\mathrm{mL}) \times 0.1 \mathrm{NaOH}(\mathrm{M}) \times 0.07 \times 100}{10 \mathrm{~g}} \times 100 \%
$$

\section{Ascorbic acid content}

Acetic acid solution (50 mL, 1\%) was vortex mixed with sliced mango pulp $(10 \mathrm{~g})$. The mixture was then centrifuged at $5000 \times$ $g$ for $15 \mathrm{~min}$ to separate the supernatant. An aliquot of the supernatant $(10 \mathrm{~mL})$ was titrated with 2,6-dichlorophenolindophenol to a reddish color, with the titration volume recorded when the color was stable for at least $30 \mathrm{~s}$. Acetic acid solution $(10 \mathrm{~mL}, 1 \%)$ was used as the blank control, with titration performed as detailed above. ${ }^{28}$ The ascorbic acid content $(\mathrm{mg} / 100 \mathrm{~g})$ was calculated as follows:

Ascorbic acid $(\mathrm{mg} / 100 \mathrm{~g})=\left[\left(V_{1}-V_{2}\right) \times V_{\mathrm{T}} \times M \times 100\right] /(V \times W)$

where $V_{1}$ is the titration volume for the sample $(\mathrm{mL}), V_{2}$ is the titration volume for the blank control $(\mathrm{mL}), V_{\mathrm{T}}$ is the total supernatant volume $(\mathrm{mL}), M$ is the $V_{\mathrm{c}}$ content titrated by $1 \mathrm{~mL}$ of 2,6-dichlorophenol-indophenol, $V$ is the volume of the tested sample $(\mathrm{mL})$, and $W$ is the sample weight $(\mathrm{g})$.

\section{Catalase and peroxidase activity}

Total peroxidase (POD) and catalase (CAT) activities were assayed as previously described. ${ }^{28}$ CAT activities were 
determined by monitoring the enzyme-catalyzed decomposition of $\mathrm{H}_{2} \mathrm{O}_{2}$ by potassium permanganate. One unit of CAT activity was defined as the amount of $\mathrm{H}_{2} \mathrm{O}_{2}$ (in mol) depleted per min at $240 \mathrm{~nm}$. POD activity was measured based on the determination of guaiacol oxidation by $\mathrm{H}_{2} \mathrm{O}_{2}$ at $470 \mathrm{~nm}$.

\section{$\mathrm{H}_{2} \mathrm{O}_{2}$ content}

Mango pulp ( $1 \mathrm{~g}$ ) was ground with liquid nitrogen and then phosphate buffer $(10 \mathrm{~mL}, \mathrm{pH} 7.4)$ was added at $0{ }^{\circ} \mathrm{C}$. The mixture was centrifuged at $9000 \times g$ for $15 \mathrm{~min}$. The $\mathrm{H}_{2} \mathrm{O}_{2}$ content was determined using a commercial kit (Jiancheng Bioengineering Institute, Nanjing, China).

\section{Statistical analysis}

Data were expressed as the mean \pm standard deviation (SD) of triplicates. The least significant difference (LSD), Duncan's multiple range test, and one-way analysis of variance (ANOVA) were used for multiple comparisons and performed using SPSS 16.0 software. Differences were considered statistically significant if $p<0.05$.

\section{Conclusions}

A bacterial laccase, fmb-rL103, has been used for the first time to catalyze FA grafting onto CS. When the reaction was performed at $\mathrm{pH} 6.5$ and $60^{\circ} \mathrm{C}$ for $1 \mathrm{~h}$ with an FA concentration of $10 \mathrm{mM}$, the optimum grafting efficiency was achieved. UV-vis and FT-IR analysis confirmed the covalent grafting reaction. The antioxidant and antibacterial properties of FA- $g$-CS were significantly improved. Furthermore, coating with FA- $g$-CS significantly improved the physiological and biochemical characteristics of mango compared with the CSs coating. Therefore, an effective approach to synthesizing FA- $g$-CS using bacterial laccase has been developed in the present study, and the catalyzed products have promising applications in fruit preservation.

\section{Conflicts of interest}

There are no conflicts to declare.

\section{Acknowledgements}

This work was supported by the Fundamental Research Funds for the Central Universities (KYZ201418), and the National Natural Science Foundation of China (31201423). We thank Simon Partridge, PhD, from Liwen Bianji, Edanz Editing China (http:/www.liwenbianji.cn/ac), for editing the English text of a draft of this manuscript.

\section{References}

1 P. K. Dutta, S. Tripathi and G. K. Mehrotra, Food Chem., 2009, 114, 1173-1182.

2 M. Xie, B. Hu, Y. Wang and X. Zeng, J. Agric. Food Chem., 2014, 62, 9128-9136.
3 S. Kobayashi, H. Uyama and S. Kimura, Chem. Rev., 2001, 101, 3793-3818.

4 G. M. Gübitz and A. C. Paulo, Curr. Opin. Biotechnol., 2003, 14, 577-582.

5 C. A. Hsu, T. N. Wen and Y. C. Su, Environ. Sci. Technol., 2012, 46, 5109-5117.

6 A. Aljawish, I. Chevalot, B. Piffaut, C. Rondeau-Mouro, M. Girardin, I. Jasniewski, J. Scher and L. Muniglis, Carbohydr. Polym., 2012, 87, 537-544.

7 A. Aljawish, I. Chevalot, J. Jasniewski, A.-M. Revol-Junelles, J. Scher and L. Muniglia, Food Chem., 2014, 161, 279-287.

8 M. Božič, S. Gorgieva and V. Kokol, Carbohydr. Polym., 2012, 87, 2388-2398.

9 W. Z. Qiu, Q. Z. Zhong, Y. Du, Y. Lv and Z. K. Xu, Green Chem., 2016, 18, 6205-6208.

10 J. Sun, M. Zheng, Z. Lu, F. Lu and C. Zhang, Process Biochem., 2017, 55, 77-84.

11 J. Ulloa, G. Guatemala, E. Arriola, H. Escalona and L. Díaz, J. Food Eng., 2009, 91, 211-216.

12 P. Jongsri, T. Wangsomboondee, P. Rojsitthisak and K. Seraypheap, LWT-Food Sci. Technol., 2016, 73, 28-36.

13 E. Baldwin, M. Nisperos, R. Hagenmaier and R. Baker, Food Technol., 1997, 51, 56-62.

14 N. H. Banks, Hortic. Rev., 2002, 26, 161.

15 R. A. Muzzarelli, F. Tanfani, M. Emanuelli and S. Mariotti, Carbohydr. Res., 1982, 107, 199-214.

16 F. Sousa, G. M. Guebitz and V. Kokol, Process Biochem., 2009, 44, 749-756.

17 R. A. Muzzarelli, G. Littarru, C. Muzzarelli and G. Tosi, Carbohydr. Polym., 2003, 53, 109-115.

18 M. N. R. Kumar, React. Funct. Polym., 2000, 46, 1-27.

19 K. T. Dang, Z. Singh and E. E. Swinny, J. Agric. Food Chem., 2008, 56, 1361-1370.

20 M. Cissé, J. Polidori, D. Montet, G. Loiseau and M. N. Ducamp-Collin, Postharvest Biol. Technol., 2015, 101, 10-14.

21 Y. Kim, J. K. Brecht and S. T. Talcott, Food Chem., 2007, 105, 1327-1334.

22 E. Baldwin, J. Burns, W. Kazokas, J. Brecht, R. Hagenmaier, R. Bender and E. Pesis, Postharvest Biol. Technol., 1999, 17, 215-226.

23 P. Trotel-Aziz, M. Couderchet, G. Vernet and A. Aziz, Eur. J. Plant Pathol., 2006, 114, 405-413.

24 G. A. Gonzalez-Aguilar, J. A. Villa-Rodriguez, J. F. AyalaZavala and E. M. Yahia, Trends Food Sci. Technol., 2010, 21, 475-482.

25 X. Meng, B. Li, J. Liu and S. Tian, Food Chem., 2008, 106, 501508.

26 S. Sabnis and L. H. Block, Int. J. Biol. Macromol., 2000, 27, 181-186.

27 Y. Yu, S. Zhang, Y. Ren, H. Li, X. Zhang and J. Di, J. Food Eng., 2012, 113, 4085-4414.

28 F. Razavi and J. Hajilou, Sci. Hortic., 2016, 200, 95-101.

29 P. Jongsri, T. Wangsomboondee, P. Rojsitthisak and K. Seraypheap, LWT-Food Sci. Technol., 2016, 73, 28-36. 